\title{
Incentivizing Prosumer Coalitions with Energy Management using Cooperative Game Theory
}

\author{
Liyang Han, Student Member, IEEE, Thomas Morstyn, Member, IEEE, \\ and Malcolm McCulloch, Senior Member, IEEE
}

\begin{abstract}
The advances in distributed renewable generation technologies in recent years have started to cause load balancing issues in power networks. Distributed energy storage (ES) systems, although seen as a tool to mitigate the stress on local networks, tend to be operated only to minimize the energy cost of their direct owner. In this paper, cooperative game theory is used to construct an energy grand coalition, in which ES system operations are optimized to minimize the coalitional energy cost. Case studies then show that forming energy coalitions is effective in reducing the variability of a local network load profile. The resulting cooperative game is mathematically proven to be balanced, which means the energy cost savings from the cooperative ES operation can be allocated to the players within the energy grand coalition in a manner that disincentivizes them from exiting from the grand coalition to form smaller coalitions.
\end{abstract}

Index Terms-Cooperative game theory, energy management, energy storage, linear programming, nucleolus, prosumers

\section{INTRODUCTION}

$\mathbf{T}$ HIS paper explores the use of cooperative game theory in a distribution network of prosumers, proactive-consumers with distributed energy resources that actively control their energy behaviors. Through collaborative energy management of each prosumer's energy storage (ES) system, it is shown that not only can prosumers profit from this cooperative scheme, but the distribution network can benefit from the resulting reduced peak power flows and smoothed load profiles.

The adoption of energy resources in the electricity distribution network (e.g. distributed solar PV) is a by-product of the rapid renewable technological development and energy regulations favoring renewable energy since the late 20th century [1]. The emergence of prosumers changed the traditionally unidirectional power network to a bidirectional one. This resulted in two issues: 1) power supply unreliability due to the variability of intermittent distributed generations [2], and 2) difficulties in voltage control due to high reverse power flows [3]. Energy storage (ES) systems are widely seen as a solution to these problems as they can quickly respond to the generation and load imbalance in a power network and provide mitigation when needed [4].

Based on the architecture, ES control strategies can be largely divided into three categories: centralized, decentral-

Manuscript received December 4, 2017; revised May 14, 2018; accepted July 8, 2018; date of current version July 19, 2018.

This work was supported in part by the Engineering and Physical Sciences Research Council under Grants EP/N03466X/1 and EP/S000887/1, and in part by the Oxford Martin Programme on Integrating Renewable Energy.

The authors are with the Department of Engineering Science, University of Oxford, Oxford, United Kingdom (e-mail: liyang.han@eng.ox.ac.uk; thomas.morstyn@eng.ox.ac.uk; malcolm.mcculloch@eng.ox.ac.uk). ized, and distributed [5]. A centralized control strategy has scalability issues but can serve as the benchmark for the best possible performance [6]. Various decentralized and distributed control strategies have been proposed in microgrid applications [7]-[10], but they are inevitably limited by the assumption that all of the participants align their interests with the microgrid or the grid [11]. We recognize, however, that prosumers tend to be profit-driven, and will not change their energy behavior altruistically for the benefit of the grid with no or negative financial gain for themselves. Therefore, we identify game theory as a methodology to connect the ES control strategies of different rational entities whose decisions affect one another's economic outcomes. In fact, both noncooperative game theory and cooperative game theory are adopted in a wide range of studies concerning energy management [12].

Noncooperative game theory is often used to study prosumer strategic behaviors. One key area of research concerning EV charging revolves around "valley filling" [13] by solving for the Nash equilibrium [14], where EV owners operate their EV batteries to maximize their own welfare [15]-[17] under dynamic pricing. In recent literature, noncooperative game theory is often used in energy sharing among prosumers [18][20], who are expected to strategically adjust their energy behavior according to the dynamic dual prices set by either a for-profit retailer [18], or a non-profit aggregator such as a microgrid operator (MGO) [19], or a multi-energy system (MES) [20]. A Stackelberg game approach is used in [18] to analyze the trade-off between the retail profit and the consumer surplus, which is then extended by [19] to a bilevel optimization problem for a microgrid. Natural gas and heat energy is further included in [20] in addition to electricity.

Two caveats can be identified with the usage of noncooperative game theory in prosumer energy sharing. First, optimization dual price variables can be interpreted as competitive energy prices, and it heavily relies on the assumption that no prosumers have market power [21]. Second, the allocation of the financial benefit to each prosumer is either unclear or suboptimal on the individual level. In other words, it might be possible for a subgroup of participants to abandon the current scheme and develop an alternative that are economically preferable. Virtual power plants [22], price-based peerto-peer energy trading [23], and the framework of alternating direction method of multipliers (ADMM) [24] are proposed as alternative energy sharing schemes, but they have similar drawbacks as the noncooperative game theoretic approaches because they also rely on prosumers being price takers within a pre-defined group. 
Cooperative game theory, on the other hand, incentivizes independent decision makers to act together, investigates the fairness of profit allocation from each individual player's perspective and gives players the flexibility to decide with whom to cooperate [25]. It has been used to study ways of reducing distribution losses [26], and increasing the generation bidding price through aggregation [27]. In [28], a direct electricity trading scheme is proposed to allow small-scale local trading, where a higher joint revenue is shown using cooperative game theory, but it does not consider any load shifting mechanisms including ES operation.

The novelty of this paper lies in its intersection of cooperative game theory and energy management optimization. Proposed is a local energy coalition among prosumers, where ES systems operate collaboratively under a centralized control to minimize the joint coalitional energy cost. Cooperative game theory is used to develop a profit sharing scheme, which not only ensures that all participants are financially rewarded, but also discourages participants from deviating from the expected cooperation. This collaborative approach is then shown through case studies to provide services such as peak shaving and energy shifting to increase local utilization of renewable energy.

The rest of this paper is organized as follows. Section II introduces the concept of coalitional energy management, along with major assumptions and mathematical operators. Section III introduces cooperative game theory as the methodology to structure the economic payoffs for the coalition participants. Section IV explains in detail the model implementation, including the optimization of coalitional ES operation and the computation of the payoffs. Section V uses case studies to demonstrate the economic payback to the participants and the benefit on the power network under the proposed strategy. Section VI concludes the paper.

\section{Coalitional Energy Management}

For a prosumer who owns a distributed PV system and an ES system, the common objective in scheduling the ES operation is to minimize the energy cost. Because the retail supplier normally offers a lower export tariff (e.g. feed-in tariff) than it charges for buying electricity, prosumers can save money by storing the excess PV generation in the ES system and using it when the generation is less than they need.

If we consider a group of prosumers who have different load profiles and distributed energy resources, when they each manage their own ES system to minimize their energy bill, the joint operation of all the ES systems is unlikely to yield the minimum total energy cost for the group. However, it is possible for all the ES systems to cooperate to minimize the total group energy cost, the additional savings from which can then be allocated among the prosumers.

\section{A. Prosumer Coalitional Energy Cost}

Let a group of $N$ prosumers form grand coalition $\mathcal{N}$ indexed by $i \in \mathcal{N}:=\{1,2, \ldots, N\}$. For each prosumer $i$ we assume no more than one ES system. We then consider $K$ timesteps $(t=1,2, \ldots, K)$ with a time interval of $\Delta t$. We define the following vectors for each timestep $t$ :

$\mathbf{q}_{i} \in \mathbb{R}^{K \times 1}$ : Prosumer $i$ 's net energy consumption (positive) and generation (negative) without ES, $[k W h]$

$\mathbf{p}_{b} \in \mathbb{R}^{K \times 1}$ : Electricity buy price, $[£ / k W h]$

$\mathbf{p}_{s} \in \mathbb{R}^{K \times 1}$ : Electricity sell price (export tariff), $[£ / k W h]$

$\mathbf{b}_{i} \in \mathbb{R}^{K \times 1}$ : Prosumer $i$ 's ES energy charge (positive) and discharge (negative) variables, $[k W h]$

The remuneration for exporting distributed generation has been consistently declining in recent years, and this paper assumes that $\mathbf{p}_{b}>\mathbf{p}_{s}$ in every timestep, as is already the case in countries of relatively high penetrations of distributed generation such as the UK [29], [30], and Germany [31]. It is also assumed that the electricity price can vary by each timestep but are required to be known inputs before each modeling cycle (e.g. day-ahead). Line losses are not accounted for considering the small size of a local power network.

The load consumption and generation of all prosumers are assumed to be known inputs, but they are not restricted to a certain type of profile (e.g. residential, commercial, industrial, etc.). A prosumer with only an ES unit (e.g. a public energy storage unit invested by an independent entity) can be modeled with all load and generation inputs set to zero. In reality, the model will be using predicted consumption and generation data, and the impact of uncertainties and prediction errors is analyzed in the case studies.

We then construct the net energy load and ES operation variable matrices for the grand coalition:

$$
\begin{aligned}
\mathbf{Q}^{\mathcal{N}} \in \mathbb{R}^{K \times N}:=\left[\mathbf{q}_{1}, \mathbf{q}_{2}, \ldots, \mathbf{q}_{N}\right] \\
\mathfrak{B} \in \mathbb{R}^{K \times N}:=\left[\mathbf{b}_{1}, \mathbf{b}_{2}, \ldots, \mathbf{b}_{N}\right]
\end{aligned}
$$

The energy cost for the grand coalition $\mathcal{N}$ is given by

$$
\mathbf{p}_{b}^{\top}\left[\left(\mathfrak{B}+\mathbf{Q}^{\mathcal{N}}\right) \mathbf{1}^{N}\right]^{+}+\mathbf{p}_{s}^{\top}\left[\left(\mathfrak{B}+\mathbf{Q}^{\mathcal{N}}\right) \mathbf{1}^{N}\right]^{-}
$$

where $\mathbf{1}^{X} \in \mathbb{R}^{X \times 1}$ and $\mathbf{1}_{k}^{X}=1, \forall k$, and for any matrix or vector $\mathbf{Y},[\mathbf{Y}]_{m n}^{+(-)}=\max (\min )\left\{Y_{m n}, 0\right\}$.

We then define the energy cost function for any net energy load and ES operation variable matrices $\mathbf{Q}, \mathfrak{B} \in \mathbb{R}^{K \times N}$ :

$$
\begin{gathered}
F(\mathfrak{B}, \mathbf{Q})=\mathbf{p}_{b}^{\top}\left[(\mathfrak{B}+\mathbf{Q}) \mathbf{1}^{N}\right]^{+}+\mathbf{p}_{s}^{\top}\left[(\mathfrak{B}+\mathbf{Q}) \mathbf{1}^{N}\right]^{-} \\
\text {Scaling } \mathbf{Q} \text { and } \mathfrak{B} \text { by a factor of } a \geq 0, \text { we have } \\
F(a \mathfrak{B}, a \mathbf{Q})=a F(\mathfrak{B}, \mathbf{Q})
\end{gathered}
$$

\section{B. Coalitions}

A coalition is any subset $\mathcal{S} \subseteq \mathcal{N}, \mathcal{N}$ being the grand coalition. The set of all possible coalitions is defined as the power set $2^{\mathcal{N}}$ of $\mathcal{N}$. In other words, $\mathcal{S} \in 2^{\mathcal{N}}, \forall \mathcal{S} \subseteq \mathcal{N}$.

For any matrix $\mathbf{Z}^{\mathcal{N}} \in \mathbb{R}^{K \times N}:=\left[\mathbf{z}_{1}, \mathbf{z}_{2}, \ldots, \mathbf{z}_{N}\right]$, where $\mathbf{z}_{i} \in \mathbb{R}^{K \times 1}, \forall i \in[1, N]$, we define the following operations that use coalition superscripts: where $\mathcal{S}_{j} \in 2^{\mathcal{N}}$, and $a_{j} \geq 0$, indexed by $j=1,2, \ldots, 2^{N}$

1) coalition scaling

$$
\mathbf{Z}^{a_{j} \mathcal{S}_{j}}=\left[a_{j} \mathbf{z}_{1} 1_{1}^{\mathcal{S}_{j}}, a_{j} \mathbf{z}_{2} 1_{2}^{\mathcal{S}_{j}}, \ldots, a_{j} \mathbf{z}_{N} 1_{N}^{\mathcal{S}_{j}}\right]
$$

where the indicator function $1_{i}^{\mathcal{S}}=\left\{\begin{array}{ll}1, & \text { if } i \in \mathcal{S} \\ 0, & \text { otherwise }\end{array}\right.$. Thus

$$
\mathbf{Z}^{a_{j} \mathcal{S}_{j}}=a_{j}\left[\mathbf{z}_{1} 1_{1}^{\mathcal{S}_{j}}, \mathbf{z}_{2} 1_{2}^{\mathcal{S}_{j}}, \ldots, \mathbf{z}_{N} 1_{N}^{\mathcal{S}_{j}}\right]=a_{j} \mathbf{Z}^{\mathcal{S}_{j}}
$$


For a special case where $a_{j}=1, \mathcal{S}_{j}=\mathcal{S}$, we have

$$
\mathbf{Z}^{\mathcal{S}}=\left[\mathbf{z}_{1} 1_{1}^{\mathcal{S}}, \mathbf{z}_{2} 1_{2}^{\mathcal{S}}, \ldots, \mathbf{z}_{N} 1_{N}^{\mathcal{S}}\right]
$$

2) coalition summation

$$
\begin{aligned}
& \mathbf{Z}^{\sum_{j=1}^{2^{N}} a_{j} \mathcal{S}_{j}} \\
= & {\left[\sum_{j=1}^{2^{N}} a_{j} \mathbf{z}_{1} 1_{1}^{\mathcal{S}_{j}}, \sum_{j=1}^{2^{N}} a_{j} \mathbf{z}_{2} 1_{2}^{\mathcal{S}_{j}}, \ldots, \sum_{j=1}^{2^{N}} a_{j} \mathbf{z}_{N} 1_{N}^{\mathcal{S}_{j}}\right] }
\end{aligned}
$$

from which we can derive:

$$
\begin{aligned}
\mathbf{Z}^{\sum_{j=1}^{2^{N}} a_{j} \mathcal{S}_{j}} & =\sum_{j=1}^{2^{N}}\left[a_{j} \mathbf{z}_{1} 1_{1}^{\mathcal{S}_{j}}, a_{j} \mathbf{z}_{2} 1_{2}^{\mathcal{S}_{j}}, \ldots, a_{j} \mathbf{z}_{N} 1_{N}^{\mathcal{S}_{j}}\right] \\
& \stackrel{(3)}{=} \sum_{j=1}^{2^{N}} \mathbf{Z}^{a_{j} \mathcal{S}_{j}} \stackrel{(4)}{=} \sum_{j=1}^{2^{N}} a_{j} \mathbf{Z}^{\mathcal{S}_{j}}
\end{aligned}
$$

For a special case where $a_{j}=\left\{\begin{array}{ll}1, & \mathcal{S}_{j}=\mathcal{S} \text { or } \mathcal{T} \\ 0, & \text { otherwise }\end{array}\right.$, and $\mathcal{S} \cap \mathcal{T}=\emptyset$, we have $1_{i}^{\mathcal{S}}+1_{i}^{\mathcal{T}}=\mathbf{1}_{i}^{\mathcal{S} \cup \mathcal{T}}$, so

$$
\mathbf{Z}^{\mathcal{S}+\mathcal{T}}
$$$$
=\left[\mathbf{z}_{1}\left(1_{1}^{\mathcal{S}}+1_{1}^{\mathcal{T}}\right), \mathbf{z}_{2}\left(1_{2}^{\mathcal{S}}+1_{2}^{\mathcal{T}}\right), \ldots, \mathbf{z}_{N}\left(1_{N}^{\mathcal{S}}+1_{N}^{\mathcal{T}}\right)\right]
$$$$
=\left[\mathbf{z}_{1} 1_{1}^{\mathcal{S} \cup \mathcal{T}}, \mathbf{z}_{2} 1_{2}^{\mathcal{S} \cup \mathcal{T}}, \ldots, \mathbf{z}_{N} 1_{N}^{\mathcal{S} \cup \mathcal{T}}\right]=\mathbf{Z}^{\mathcal{S} \cup \mathcal{T}}
$$

Using (5), we have $\mathbf{Q}^{\mathcal{S}}=\left[\mathbf{q}_{1} 1_{1}^{\mathcal{S}}, \mathbf{q}_{2} 1_{2}^{\mathcal{S}}, \ldots, \mathbf{q}_{N} 1_{N}^{\mathcal{S}}\right]$. Notice that any column $i$ in $\mathbf{Q}^{\mathcal{S}}$ corresponding to prosumer $i$ that does not belong to $\mathcal{S}$ is set to zero. Written in the format of (1), the energy cost for $\mathcal{S}$ is

$$
F\left(\mathfrak{B}, \mathbf{Q}^{\mathcal{S}}\right)=\mathbf{p}_{b}^{\top}\left[\left(\mathfrak{B}+\mathbf{Q}^{\mathcal{S}}\right) \mathbf{1}^{N}\right]^{+}+\mathbf{p}_{s}^{\boldsymbol{\top}}\left[\left(\mathfrak{B}+\mathbf{Q}^{\mathcal{S}}\right) \mathbf{1}^{N}\right]^{-}
$$

Note that the net energy load matrix $\mathbf{Q}$ is superscripted and the ES operation variable matrix $\mathfrak{B}$ is not. This is because we assume in our model the net energy loads are known parameters, and all elements in $\mathbf{Q}^{\mathcal{S}}$ can be directly calculated by applying (5) directly to the input parameters, while $\mathfrak{B}$ is a matrix containing variables that can take on any values, which are yet to be constrained.

One of the premises of this paper is that all ES systems are fully controllable. As $\mathfrak{B}$ is the only variable in (8), we construct a coalitional energy cost function $C_{\mathcal{S}}(\mathfrak{B})$ given by

$$
C_{\mathcal{S}}(\mathfrak{B})=F\left(\mathfrak{B}, \mathbf{Q}^{\mathcal{S}}\right)
$$

Before we start minimizing $C_{\mathcal{S}}(\mathfrak{B})$, we need to consider all the ES operational constraints that $\mathfrak{B}$ is subject to, which are discussed in II-C.

\section{ES Operational Constraints}

To represent the ES operation realistically, we need to constrain the ES operation variables $\mathfrak{B}$ properly. Therefore we assign to each player's ES system an energy capacity of $e_{i} \in \mathbb{R}_{\geq 0}$, in $[k W h]$, charge and discharge limits over the time span of $\Delta t$ of $\bar{b}_{i}, \underline{b}_{i} \in \mathbb{R}_{\geq 0}$, in $[k W h]$, charge and discharge efficiencies of $\eta_{i}^{\text {in }}, \eta_{i}^{\text {out }} \in(0,1)$, state of charge upper and lower limits of $S_{0} C_{i}, S_{0} C_{i} \in[0,1]$, and an initial state of charge of $S o C_{i}^{0} \in[0,1]$. We construct

$$
\begin{aligned}
\mathbf{E}^{\mathcal{N}} \in \mathbb{R}^{K \times N} & :=\left[e_{1} \mathbf{1}^{K}, e_{2} \mathbf{1}^{K}, \ldots, e_{N} \mathbf{1}^{K}\right] \\
\overline{\mathbf{B}}^{\mathcal{N}} & \in \mathbb{R}^{K \times N}:=\left[\bar{b}_{1} \mathbf{1}^{K}, \bar{b}_{2} \mathbf{1}^{K}, \ldots, \bar{b}_{N} \mathbf{1}^{K}\right] \\
\underline{\mathbf{B}}^{\mathcal{N}} \in \mathbb{R}^{K \times N}: & :=\left[\underline{b}_{1} \mathbf{1}^{K}, \underline{b}_{2} \mathbf{1}^{K}, \ldots, \underline{b}_{N} \mathbf{1}^{K}\right] \\
\eta^{\mathbf{I}} & \in \mathbb{R}^{N \times N}:=\operatorname{diag}\left(\eta_{1}^{\text {in }}, \eta_{2}^{\text {in }}, \ldots, \eta_{N}^{\text {in }}\right) \\
\eta^{\mathbf{O}} & \in \mathbb{R}^{N \times N}:=\operatorname{diag}\left(1 / \eta_{1}^{\text {out }}, 1 / \eta_{2}^{\text {out }}, \ldots, 1 / \eta_{N}^{\text {out }}\right)
\end{aligned}
$$

$$
\begin{aligned}
\overline{\mathbf{S o C}} \in \mathbb{R}^{N \times N}:=\operatorname{diag}\left(\overline{S o C}_{1}, \overline{S o C}_{2}, \ldots, \overline{S o C}_{N}\right) \\
\underline{\mathbf{S o C}} \in \mathbb{R}^{N \times N}:=\operatorname{diag}\left(\underline{S o C_{1}}, \underline{S o C_{2}}, \ldots, \underline{S o C_{N}}\right) \\
\text { SoC }^{\mathbf{0}} \in \mathbb{R}^{N \times N}:=\operatorname{diag}\left(S o C_{1}^{0}, \underline{S o C}_{2}^{0}, \ldots, S o C_{N}^{0}\right)
\end{aligned}
$$

Using (5), we construct $\mathbf{E}^{\mathcal{S}}, \overline{\mathbf{B}}^{\mathcal{S}}$ and $\underline{\mathbf{B}}^{\mathcal{S}}$ as the energy capacity, charge limit and discharge limit matrices for $\mathcal{S}$.

An ES operation variable matrix $\mathfrak{B}^{\langle\mathcal{S}\rangle}$ is defined as a feasible ES operation matrix for $\mathcal{S}$, thus subject to:

1) Power constraint: the charge and discharge energy for each timestep is constrained by the charge and discharge limit:

$$
\underline{\mathbf{B}}^{\mathcal{S}} \leq \mathfrak{B}^{\langle\mathcal{S}\rangle} \leq \overline{\mathbf{B}}^{\mathcal{S}}
$$

Matrix inequality in this paper is established only between matrices of the same size such that the inequality specified is satisfied by every pair of elements of the same index on the two sides of the inequality sign. For example, (10) is equivalent to $\underline{B}_{t i}^{\mathcal{S}} \leq B_{t i}^{\langle\mathcal{S}\rangle} \leq \bar{B}_{t i}^{\mathcal{S}}, \forall t, \forall i$. Notice that $B_{t i}^{\langle\mathcal{S}\rangle}=0, \forall i \notin \mathcal{S}, \forall t$, because $\underline{B}_{t i}^{\mathcal{S}}=\bar{B}_{t i}^{\mathcal{S}}=0, \forall i \notin \mathcal{S}$ based on (5).

2) Energy constraint: each ES system's state of charge is limited between its upper and lower limits for every timestep:

$$
\begin{aligned}
& \mathbf{E}^{\mathcal{S}} \underline{\mathbf{S o C}} \leq \mathbf{E}^{\mathcal{S}} \mathbf{S o C}^{\mathbf{0}}+\mathbf{A}^{K}\left(\left[\mathfrak{B}^{\langle\mathcal{S}\rangle}\right]^{+} \eta^{\mathbf{I}}+\left[\mathfrak{B}^{\langle\mathcal{S}\rangle}\right]^{-} \eta^{\mathbf{O}}\right) \\
& \leq \mathbf{E}^{\mathcal{S}} \overline{\mathbf{S o C}}
\end{aligned}
$$

3) Cycle constraint: the state of charge at $t=K$ is required to be restored to $S o C_{i}^{0}$ for every ES system:

$$
\left(\mathbf{1}^{K}\right)^{\boldsymbol{\top}}\left(\left[\mathfrak{B}^{\langle\mathcal{S}\rangle}\right]^{+} \eta^{\mathbf{I}}+\left[\mathfrak{B}^{\langle\mathcal{S}\rangle}\right]^{-} \eta^{\mathbf{O}}\right)=\mathbf{0}
$$

Constraints 1-3 are denoted by $G\left(\mathfrak{B}^{\langle\mathcal{S}\rangle}, \mathbf{E}^{\mathcal{S}}, \underline{\mathbf{B}}^{\mathcal{S}}, \overline{\mathbf{B}}^{\mathcal{S}}\right)=1$, where $G\left(\mathfrak{B}, \mathbf{E}^{\mathcal{S}}, \underline{\mathbf{B}}^{\mathcal{S}}, \overline{\mathbf{B}}^{\mathcal{S}}\right)$ with $\mathfrak{B}$ as the variable is defined

$G\left(\mathfrak{B}, \mathbf{E}^{\mathcal{S}}, \underline{\mathbf{B}}^{\mathcal{S}}, \overline{\mathbf{B}}^{\mathcal{S}}\right)= \begin{cases}1, & \text { constraints } 1-3 \text { are all satisfied. } \\ 0, & \text { otherwise. }\end{cases}$

Lemma 1: $G\left(a \mathfrak{B}^{\langle\mathcal{S}\rangle}, a \mathbf{E}^{\mathcal{S}}, a \underline{\mathbf{B}}^{\mathcal{S}}, a \overline{\mathbf{B}}^{\mathcal{S}}\right)=1, \forall a>0$.

Proof: See Appendix A.

The ES model used in this paper can be used to represent a range of ES devices as well as flexible loads and demand response, which generally require a certain amount of energy to be provided during certain periods of time. Modeling a variety of demand side flexible services with a linear model is a common practice in smart grid applications [32].

\section{Optimal ES Operation}

To compute the optimal ES operation we construct functional $\theta$ given by

$$
\begin{aligned}
\theta[\mathcal{Y}] & =\min _{\mathfrak{B} \in \mathbb{R}^{K \times N}} F\left(\mathfrak{B}, \mathbf{Q}^{\mathcal{Y}}\right) \text { s.t. } G\left(\mathfrak{B}, \mathbf{E}^{\mathcal{Y}}, \underline{\mathbf{B}}^{\mathcal{Y}}, \overline{\mathbf{B}}^{\mathcal{Y}}\right)=1 \\
& =\min _{\mathfrak{B} \in \mathfrak{O}_{\mathcal{Y}}} F\left(\mathfrak{B}, \mathbf{Q}^{\mathcal{Y}}\right)
\end{aligned}
$$

where $\mathcal{Y}=\left\{\sum_{j=1}^{2^{N}} a_{j} \mathcal{S}_{j} \mid \mathcal{S}_{j} \in 2^{\mathcal{N}}, a_{j} \geq 0\right\}$, and $\mathfrak{D}_{\mathcal{Y}}=$ $\left\{\mathfrak{B} \in \mathbb{R}^{K \times N} \mid G\left(\mathfrak{B}, \mathbf{E}^{\mathcal{Y}}, \underline{\mathbf{B}}^{\mathcal{Y}}, \overline{\mathbf{B}}^{\mathcal{Y}}\right)=1\right\}$. Notice that for $\mathcal{S} \in 2^{\mathcal{N}}, \theta[\mathcal{S}]$ represents its minimum coalitional energy cost.

Lemma 2: $\theta\left[\sum_{j=1}^{2^{N}} a_{j} \mathcal{S}_{j}\right]=\theta\left[\sum_{i \in \mathcal{N}} \sum_{j=1}^{2^{N}} a_{j} 1_{i}^{\mathcal{S}_{j}}\{i\}\right]$, where $\mathcal{S}_{j} \in 2^{\mathcal{N}}, a_{j} \geq 0$.

Proof: See Appendix B. 
We denote $\mathfrak{B}^{\langle\mathcal{Y}\rangle *}$ as the optimal decision variable matrix to (13), so we have

$$
C_{\mathcal{Y}}\left(\mathfrak{B}^{\langle\mathcal{Y}\rangle *}\right)=F\left(\mathfrak{B}^{\langle\mathcal{Y}\rangle *}, \mathbf{Q}^{\mathcal{Y}}\right)=\theta[\mathcal{Y}]
$$

The minimization process provides that

$$
C_{\mathcal{Y}}\left(\mathfrak{B}^{\langle\mathcal{Y}\rangle *}\right) \leq C_{\mathcal{Y}}\left(\mathfrak{B}^{\langle\mathcal{Y}\rangle}\right), \forall \mathfrak{B}^{\langle\mathcal{Y}\rangle} \in \mathfrak{O}_{\mathcal{Y}}
$$

Lemma 3: For $\mathcal{S}, \mathcal{T} \in 2^{\mathcal{N}}$ and scalars $a, b \geq 0$, the functional $\theta$ has the following properties:

1) (Positive Homogeneity) $\theta[a \mathcal{S}]=a \theta[\mathcal{S}]$, from which we can also prove: $\theta[a \mathcal{S}]+\theta[b \mathcal{S}]=\theta[(a+b) \mathcal{S}]$.

2) (Subadditivity) $\theta[a \mathcal{S}]+\theta[b \mathcal{T}] \geq \theta[a \mathcal{S}+b \mathcal{T}]$

Proof: See Appendix C.

\section{iII. Prosumer Cooperative Game Formulation}

A cooperative game analyzes situations where additional values can be achieved when players operate collaboratively. Here, we apply the standard way of analyzing a cooperative game on the $N$-prosumer energy coalition we constructed.

\section{A. Value of Energy Coalitions}

For each energy coalition $\mathcal{S}$ we specify a characteristic function or value function $v(\mathcal{S}): 2^{\mathcal{N}} \rightarrow \mathbb{R}$ that represents the value of $\mathcal{S}$. We define this value as the energy cost savings by forming $\mathcal{S}$, which is the difference between the sum of the minimum energy costs of each prosumer in $\mathcal{S}$ by optimizing their ES systems individually, and the minimum coalitional energy cost of $\mathcal{S}$ by optimizing the ES systems cooperatively:

$$
v(\mathcal{S})=\sum_{i \in \mathcal{S}} C_{\{i\}}\left(\mathfrak{B}^{\langle\{i\}\rangle *}\right)-C_{\mathcal{S}}\left(\mathfrak{B}^{\langle\mathcal{S}\rangle *}\right)
$$

The pair $(\mathcal{N}, v)$ defines our prosumer cooperative game, and the value of the grand coalition $v(\mathcal{N})$ denotes the total amount of payoffs we can award to the prosumers.

\section{B. Core of the Prosumer Cooperative Game}

Having calculated the total amount of payoff available, the next step is to determine how to allocate it to each prosumer.

Definition 1 (Imputation): We use vector $\mathbf{x} \in \mathbb{R}^{1 \times N}$ as the payoff allocation whose entry $x_{i}$ represents the payment to prosumer $i \in \mathcal{N}$. $\mathbf{x}$ is said to be an imputation if it meets both the Efficiency and Individual Rationality criteria:

1) (Efficiency) A payoff allocation $\mathrm{x}$ is said to be efficient if $\sum_{i \in \mathcal{N}} x_{i}=v(\mathcal{N})$, meaning the whole amount of the grand coalition's energy cost savings is allocated to the prosumers.

2) (Individual Rationality) A payoff allocation $\mathbf{x}$ is said to be individually rational if $x_{i} \geq v(\{i\}), \forall i \in \mathcal{N}$. In our game, because the value function represents the energy cost savings, $v(\{i\})=0, \forall i \in \mathcal{N}$. Therefore, this criterion guarantees that no prosumer is losing money by joining the grand coalition. This criterion is very important because it discourages strategic behaviors and protects players from the possibility of being financially disadvantaged if other players who indeed try to exercise their market power.

The set of all imputations for our game is defined as

$$
\mathcal{I}:=\left\{\mathbf{x} \in \mathbb{R}^{1 \times N} \mid \sum_{i \in \mathcal{N}} x_{i}=v(\mathcal{N}), x_{i} \geq v(\{i\}), \forall i \in \mathcal{N}\right\}
$$

However, an imputation does not guarantee everyone being satisfied in the grand coalition, as some players may be able to achieve higher payoffs by forming smaller energy coalitions.
Definition 2 (Excess): We measure an energy coalition's dissatisfaction with respect to the imputation $\mathrm{x}$ by its excess defined as $\varepsilon(\mathbf{x}, \mathcal{S})=v(\mathcal{S})-\sum_{i \in \mathcal{S}} x_{i}$.

When $\varepsilon(\mathbf{x}, \mathcal{S})>0$, it indicates that $\mathcal{S}$ breaking off from the grand coalition can offer higher payoffs to its members.

Definition 3 (Core): For our cooperative game $(\mathcal{N}, v)$, the core $\mathcal{C}$ is defined as the set of imputations such that no energy coalition has a value greater than the sum of its members' allocated payoffs: $\mathcal{C}:=\left\{\mathbf{x} \in \mathcal{I} \mid \varepsilon(\mathbf{x}, \mathcal{S}) \leq 0, \forall \mathcal{S} \in 2^{\mathcal{N}}\right\}$.

A payoff allocation $\mathrm{x}$ is said to be stabilizing if $\mathrm{x} \in \mathcal{C}$. However, some games have empty cores, meaning no matter how payoffs are allocated, there will always be one or more coalitions that are dissatisfied.

\section{Shapley Value vs. Nucleolus}

Two important classes of games with nonempty cores are convex games and balanced games [27].

Definition 4 (Convex Game): A game is said to be convex if $v(\mathcal{S})+v(\mathcal{T}) \leq v(\mathcal{S} \cup \mathcal{T})+v(\mathcal{S} \cap \mathcal{T}), \forall \mathcal{S}, \mathcal{T} \in 2^{\mathcal{N}}$, which can be rewritten as $v(\mathcal{S} \cup\{i\})-v(\mathcal{S}) \leq v(\mathcal{T} \cup\{i\})-v(\mathcal{T})$, $\forall i \in \mathcal{N}, \forall \mathcal{S} \subset \mathcal{T} \subset \mathcal{N} \backslash\{i\}$.

This indicates an increasing marginal value for coalition members as the coalition grows in size. The core of a convex game is not empty, and one interesting imputation that is guaranteed in a convex game's core is the Shapley value [33]:

Definition 5 (Shapley Value): The Shapley value of a cooperative game is given by

$$
\phi_{i}(v)=\sum_{\mathcal{S} \in 2^{\mathcal{N}}, i \in \mathcal{S}} \frac{(|\mathcal{S}|-1) !(N-|\mathcal{S}|) !}{N !}[v(\mathcal{S})-v(\mathcal{S} \backslash\{i\})]
$$

The Shapley value also satisfies the following axioms:

1) (Efficiency) $\sum_{i \in \mathcal{N}} \phi_{i}(v)=v(\mathcal{N})$.

2) (Individual Rationality) $\phi_{i}(v) \geq v(\{i\}), \forall i \in \mathcal{N}$.

3) (Symmetry) If $v(\mathcal{S} \cup\{i\})=v(\mathcal{S} \cup\{j\}), \forall \mathcal{S} \in 2^{\mathcal{N}}, \mathcal{S} \cap$ $\{i, j\}=\emptyset$, then $\phi_{i}(v)=\phi_{j}(v)$.

4) (Dummy Axiom) If $v(\mathcal{S})=v(\mathcal{S} \cup\{i\}), \forall \mathcal{S} \in 2^{\mathcal{N}}, \mathcal{S} \cap$ $\{i\}=\emptyset$, then $\phi_{i}(v)=0$.

5) (Additivity) If $v$ and $u$ are characteristic functions, then $\phi_{i}(v+u)=\phi_{i}(v)+\phi_{i}(u), \forall i \in \mathcal{N}$.

Despite all the desirable properties the Shapley value holds, it is not in the core of our prosumer cooperative game, which is shown in a counterexample in Section V. Therefore, we continue to look at balanced games, another type of game that is guaranteed to have a nonempty core [34]:

Definition 6 (Balanced Game): A map $\alpha: 2^{\mathcal{N}} \rightarrow[0,1]$ is called a balanced map if $\sum_{\mathcal{S} \in 2^{\mathcal{N}}} \alpha(\mathcal{S}) 1_{i}^{\mathcal{S}}=1, \forall i \in \mathcal{N}$.

A cooperative game is said to be balanced if for any balanced map $\alpha$,

$$
\sum_{\mathcal{S} \in 2^{\mathcal{N}}} \alpha(\mathcal{S}) v(\mathcal{S}) \leq v(\mathcal{N})
$$

Theorem 1: The cooperative game $(\mathcal{N}, v)$ for local prosumer energy sharing is balanced.

Proof: See Appendix D.

Definition 7 (Nucleolus): We denote $\epsilon(\mathbf{x}) \in \mathbb{R}^{2^{N}-2}$ as the vector whose entries are the excesses in respect to imputation $\mathrm{x}$ for all coalitions (excluding the grand coalition) arranged in a non-increasing order. The nucleolus $\nu$ is an imputation such that $\epsilon_{j}(\nu) \leq \epsilon_{j}(\mathbf{x}), \forall \mathbf{x} \in \mathcal{I}, \forall j \in 2^{N}-2$. 
This means that the nucleolus of a cooperative game is the lexicographically minimal imputation that minimizes the dissatisfaction of the players [27]. The nucleolus always exists, and for a game with a nonempty core (balanced), the nucleolus always belongs to the core [35], hence is a stabilizing imputation.

\section{Prosumer Cooperative Model Implementation}

To implement this prosumer cooperative model, we

1) Collect model inputs, including the number of prosumers, each prosumer's load and generation profiles, ES information specified in Section II-C, and the electricity buy and sell prices.

2) Generate a list of all possible energy coalitions $\left(2^{\mathcal{N}}\right)$, and optimize the coalitional ES operation to minimize each coalitional energy cost, calculate the value of each energy coalition using (16). This step is further explained in Section IV-A.

3) Compute the desired imputation (e.g. Shapley value, nucleolus) using the coalition values calculated in the previous step, and examine its excesses to determine whether the imputation is stabilizing. This step is further explained in Section IV-B.

Proposed is a centralized ES control scheme, which would require participants to share high-level information about their energy usage and ES system specifications with the central aggregator. Data privacy is an important subject in implementing smart grid technologies, but is out of the scope of this paper.

\section{A. Energy Management Optimization}

In the optimal ES operation problem defined in (13), for energy coalition $\mathcal{S}$, the variables are $\mathcal{S}$ 's ES charge/discharge energies at each timestep denoted by $\mathfrak{B}$, subject to the power constraint, the energy constraint, and the cycle constraint, and the objective is to minimize the coalitional energy cost $C_{\mathcal{S}}(\mathfrak{B})$.

To obtain a linear energy management optimization problem, a piecewise linear ES model with constant charge and discharge efficiencies is commonly used [36]. In this paper, we define the discharge and charge energies as $\mathfrak{B}_{-}$and $\mathfrak{B}_{+}$ respectively, to avoid using the binary variable [37] while still maintaining the model's linearity.

Similarly, there is a piecewise function in the objective function due to the different buy and sell prices for electricity, so we rewrite the coalitional net load into a positive part $\mathbf{L}_{+}$ and a non-positive part $\mathbf{L}_{-}$, adapting a method from [38].

The energy management optimization problem can be rewritten in a linear format:

$$
\begin{aligned}
\min _{\substack{\mathfrak{B}_{+}, \mathfrak{B}_{-}, \mathbf{L}_{+}, \mathbf{L}_{-}}} & \mathbf{p}_{b}^{\top}\left(\mathbf{L}_{+} \mathbf{1}^{N}\right)+\mathbf{p}_{s}^{\top}\left(\mathbf{L}_{-} \mathbf{1}^{N}\right) \\
\text { s.t. } & \mathfrak{B}_{+}, \mathfrak{B}_{-}, \mathbf{L}_{+}, \mathbf{L}_{-} \in \mathbb{R}^{K \times N} \\
& \mathbf{0} \leq \mathbf{L}_{+} \\
& \mathfrak{B}_{+}+\mathfrak{B}_{-}+\mathbf{Q}^{\mathcal{S}} \leq \mathbf{L}_{+} \\
& \mathfrak{B}_{+}+\mathfrak{B}_{-}+\mathbf{Q}^{\mathcal{S}}=\mathbf{L}_{+}+\mathbf{L}_{-} \\
& \mathbf{0} \leq \mathfrak{B}_{+} \leq \overline{\mathbf{B}}^{\mathcal{S}}
\end{aligned}
$$

$$
\begin{aligned}
& \underline{\mathbf{B}}^{\mathcal{S}} \leq \mathfrak{B}_{-} \leq \mathbf{0} \\
& \mathbf{E}^{\mathcal{S}} \underline{\mathbf{S o C}} \leq \mathbf{E}^{\mathcal{S}} \mathbf{S o C}^{\mathbf{0}}+\mathbf{A}^{K}\left(\mathfrak{B}_{+} \eta^{\mathbf{I}}+\mathfrak{B}_{-} \eta^{\mathbf{O}}\right) \\
& \leq \mathbf{E}^{\mathcal{S}} \overline{\mathbf{S o C}} \\
&\left(\mathbf{1}^{K}\right)^{\top}\left(\mathfrak{B}_{+} \eta^{\mathbf{I}}+\mathfrak{B}_{-} \eta^{\mathbf{O}}\right)=\mathbf{0}
\end{aligned}
$$

Constraints (21), (22) and the minimization objective function effectively set $\mathbf{L}_{+}=\max \left\{\mathbf{0}, \mathfrak{B}_{+}+\mathfrak{B}_{-}+\mathbf{Q}^{\mathcal{S}}\right\}$, which, along with (23), sets $\mathbf{L}_{-}=\min \left\{\mathbf{0}, \mathfrak{B}_{+}+\mathfrak{B}_{-}+\mathbf{Q}^{\mathcal{S}}\right\}$. The new power constraint is denoted by (24) and (25), the new energy constraint (26), and the new cycle constraint (27). Due to the charge, discharge efficiencies $(<1)$ and the cost minimization, the optimization process will prevent $\mathfrak{B}_{+}$and $\mathfrak{B}_{-}$from both taking on non-zero values at the same time.

\section{B. Computation of Shapley Value and Nucleolus}

After a complete list of coalition values is generated using (16), the Shapley value can be easily calculated using (17).

To solve for the nucleolus of our prosumer cooperative game, we follow the iterative process below:

1) Solve the following linear optimization:

$$
\begin{aligned}
L P_{1}: \varepsilon_{1}= & \min _{\mathbf{x}, \varepsilon} \varepsilon \\
\text { s.t. } & \sum_{\forall i \in \mathcal{N}} x_{i}=v(\mathcal{N}) \\
& v(\mathcal{S})-\sum_{\forall i \in \mathcal{S}} x_{i} \leq \varepsilon, \forall \mathcal{S} \notin\{\emptyset, \mathcal{N}\}
\end{aligned}
$$

where Constraint (29) ensures the efficiency criterion of the imputation, Constraint (30) sets $\varepsilon$ as the largest excess for all coalitions, and the objective function (28) minimizes the largest excess by searching through possible imputations. We obtain optimal solution $\varepsilon_{1}$, and $\mathfrak{S}_{1}$, a set of coalitions that are common to all optimal solutions at $\varepsilon=\varepsilon_{1}$, or coalitions that correspond to constraints defined by (30) that are binding. $\varepsilon_{1}$ is the largest excess of the nucleolus although the nucleolus has not been completely computed by this time. Therefore, if $\varepsilon_{1} \leq 0$, the nucleolus is in the core.

2) Iteratively solve the following linear optimization until a unique optimal solution $\mathrm{x}^{*}$ is obtained. $\mathrm{x}^{*}$ then is the nucleolus of our prosumer cooperative game.

$$
\begin{aligned}
& L P_{j}: \varepsilon_{j}=\min _{\mathbf{x}, \varepsilon} \varepsilon \\
& \text { s.t. } \quad \sum_{\forall i \in \mathcal{N}}^{\mathbf{x}, \varepsilon} x_{i}=v(\mathcal{N}) \\
& \sum_{\forall i \in \mathcal{S}} x_{i}=v(\mathcal{S})-\varepsilon_{l}, \quad \forall \mathcal{S} \in \mathfrak{S}_{l}, \\
& \forall l \in[1, j-1] \\
& v(\mathcal{S})-\sum_{\forall i \in \mathcal{S}} x_{i} \leq \varepsilon, \quad \forall \mathcal{S} \notin\left\{\emptyset, \mathfrak{S}_{l}, \mathcal{N}\right\} \\
& \forall l \in[1, j-1]
\end{aligned}
$$

where $j \geq 2$, numbering each linear program solved during the iteration process. Constraint (31) ensures the efficiency criterion of the imputation, Constraint (32) fixes the excesses of the coalitions that are binding in all previous iterations, Constraint (33) 
sets $\varepsilon$ as the largest excess for all remaining coalitions in $L P_{j} . \mathfrak{S}_{l}:=\left\{\mathcal{S} \mid v(\mathcal{S})-\sum_{\forall i \in \mathcal{S}} x_{i}=\right.$ $\varepsilon_{l}, \forall$ optimal decision variable $\mathbf{x}$ in $\left.L P_{l}\right\}$, which represents the set of coalitions whose excesses are binding based on (33) in $L P_{l}$.

\section{CASE Studies}

In this section, we test our prosumer cooperative game using domestic load data measured in the Customer-Led Network Revolution trials, a UK smart grid demonstration project [39]. We select a time frame of 24 hours starting from the midnight of a sunny summer day in July. The PV generation data is simulated in PVWatts [40] using a $4 \mathrm{~kW}$, fixed 20 degree tilt residential system, and the London Gatwick solar data. The ES model is a battery with an energy capacity of $7 \mathrm{kWh}$, a maximum charge power of $3.5 \mathrm{~kW}$, a maximum discharge power of $3.2 \mathrm{~kW}$, both charge and discharge efficiencies of $95 \%$, an initial state of charge of $50 \%$, and a state of charge range of $20-95 \%$. The energy buy price follows a UK Economy 7 residential rate structure: $£ 0.072 / \mathrm{kWh}$ for midnight-7am, and $£ 0.1681 / \mathrm{kWh}$ for $7 \mathrm{am}-$ midnight [29], and the energy sell price is the UK feed-in tariff [30] fixed at $£ 0.0485 / \mathrm{kWh}$.

We demonstrate that the prosumer coalition does help reduce the variability of the load profile locally. We then compare the Shapley value and the nucleolus to show that both imputations are able to reward all players, but only the nucleolus is a stabilizing imputation. Lastly, we include the prediction errors into our model to show that the impact of uncertainty on the coalitional energy cost savings is insignificant.

\section{A. Load Balancing and Financial Reward}

For the first case study, we select 10 prosumers, 5 PV systems and 5 ES systems, which are randomly assigned to the prosumers. Fig. 1 compares three different coalitional load profiles: 1) without energy management, 2) with each ES system operating to minimize its direct owner's energy cost, and 3) with all ES systems operating cooperatively to minimize the coalitional energy cost. It can be easily seen in the graph that noncooperative ES operation can reduce the reverse power flow and load variability to an extent, but cooperative ES operation is significantly more effective.

Fig. 2 displays the monetary benefits using the nucleolus and Shapley value for each individual player within the energy grand coalition benchmarked against their overall energy costs. It can be seen that every player profits from the game. Small differences can be observed between the nucleolus and the Shapley value for each player, but the nucleolus's overall range of magnitude is larger with the small allocations being smaller (Player 3, 4, 5, 6, 8) and large ones larger (Player 1, 2, $7,9,10)$ than the Shapley value, which is a general trend in all the model results we obtained. Despite these small differences, their relative values among all the players follow a similar pattern. This is because both of the imputations reward players on a merit basis - whoever contributes more to reducing the coalitional energy cost tends to receive more in return.

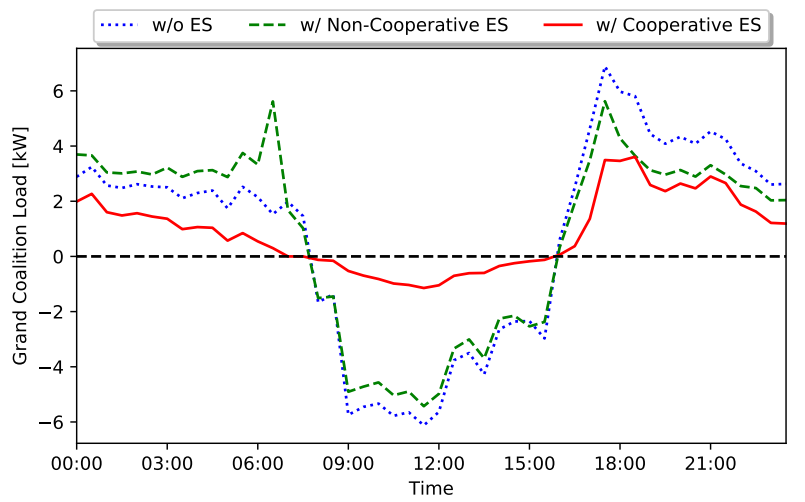

Fig. 1: 10-prosumer energy grand coalition 24-hour load profile 1) without ES, 2) with noncooperative ES, and 3) with cooperative ES

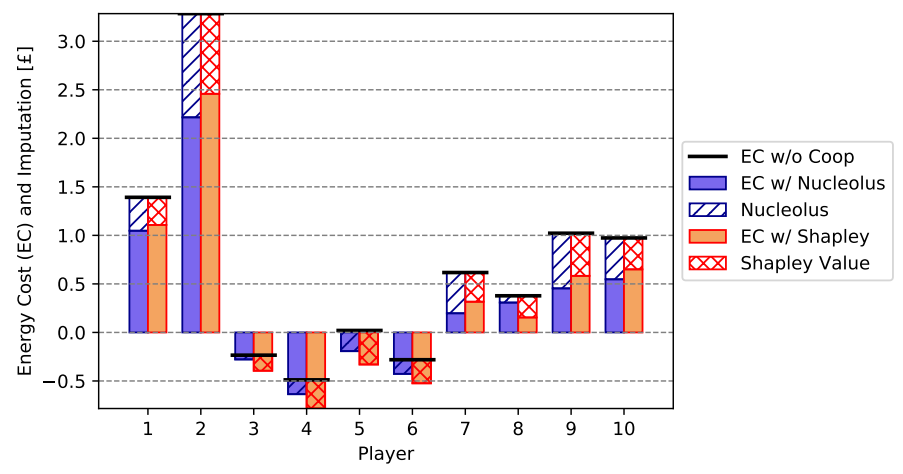

Fig. 2: 10-prosumer coalitional energy costs and cost savings over 24 hours by end user, where the baseline 'Energy Cost w/o Coop' represents the energy costs if players were to optimize their ES operation individually as opposed to cooperatively

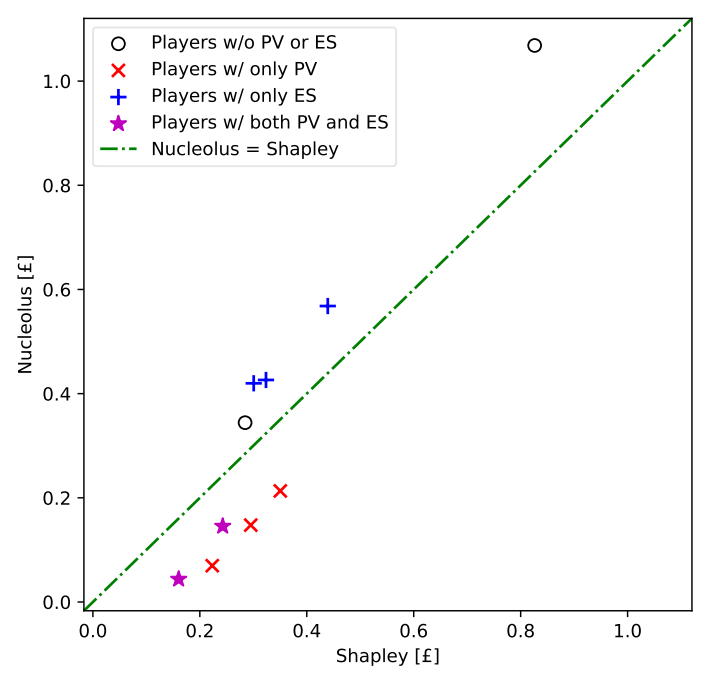

Fig. 3: Nucleolus vs. Shapley value for a 10-prosumer grand coalition

Fig. 3 shows the comparison of the nucleolus and Shapley value for each prosumer based on their resource mix. We also notice that whether a player receives more under the nucleolus or the Shapley value is dependent on its resource mix. For example, players without any PV or ES tend to be rewarded more by the nucleolus, while players with both PV and ES tend to be rewarded more by the Shapley value. This is likely due to the high penetration of PV, where pure load contributes 


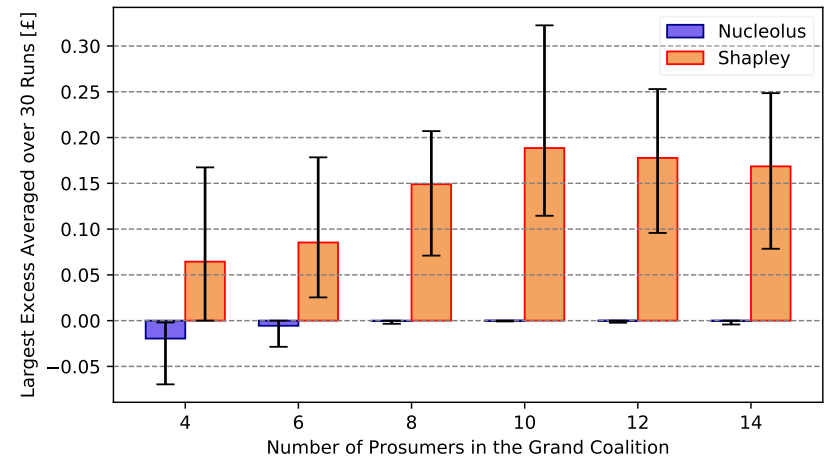

Fig. 4: Largest excesses by number of players - nucleolus vs. Shapley

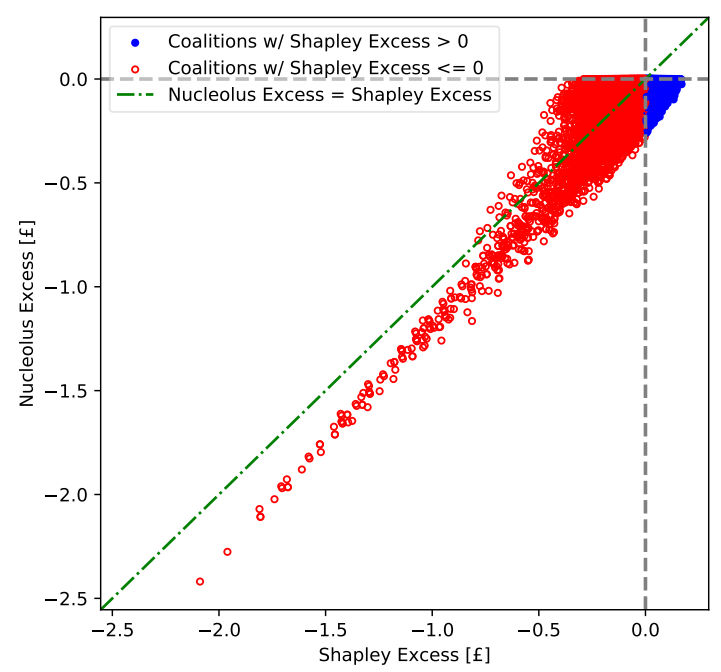

Fig. 5: Comparison of 24-hour nucleolus excesses and Shapley excesses for all coalitions within a 14-prosumer grand coalition

to higher levels of self-consumption.

\section{B. Nucleolus vs. Shapley Value}

For the second case study, we run the model with different numbers $(N=4,6,8,10,12,14)$ of players. To test a range of load profiles with different PV and ES assignments, we run the model 30 times for each number of players, and in each run, we assume 50\% ownerships of PV and ES systems respectively, all randomly assigned. We then graph the average largest excess against the number of players in Fig. 4, with the error bars showing the maximum and minimum values across all runs. It is shown that regardless of the number of prosumers in the grand coalition, the largest excess with respect to the Shapley value always exceeds zero, verifying the statement in Section III-C that the Shapley value is not in the core of our prosumer cooperative game. On the other hand, the largest excess with respect to the nucleolus is always non-positive, which validates that the nucleolus is a stabilizing imputation.

Taking the 14-prosumer game as an example, Fig. 5 maps the excesses of all the coalitions on a nucleolus vs. Shapley coordinate. The data points generally distribute along the diagonal line representing "Nucleolus Excess = Shapley Excess", but some Shapley excesses exceed zero, while no nucleolus excesses do. It is worth noting that even for the coalitions with positive Shapley excesses, their excess values split among the players in those coalitions are relatively small compared to their individual Shapley values. It is an interesting topic for future research to analyze whether this small difference is enough to drive players to leave the grand coalition to form smaller coalitions.

Similar to other centralized control strategies, the scalability of this method is limited. This is mainly because the number of linear problems solved in both the ES optimization and the nucleolus calculation is proportional to the total number of coalitions, which increases exponentially with the number of players. For instance, running the model built in Python 3.6 on an Apple iMac with a processor of $2.8 \mathrm{GHz}$ Intel Core i5 and a memory module of $16 \mathrm{~GB} 1867 \mathrm{MHz}$ DDR3, it takes an average of 15, 80 and 500 minutes for games of 12, 13, and 14 players respectively. The main goal of using a centralized control in this paper, however, is to benchmark the economic outcome with optimal ES operation [6] so as to theoretically prove the balancedness of the cooperative game structure as was done in Section III. Developing methods to improve the scalability of the model is naturally an extension of this work.

\section{The Impact of Uncertainty}

For the third case study, we conduct a Monte Carlo analysis of the impact of prediction errors on the coalitional energy cost savings. The model setup is similar to the previous case studies, except that all input load and PV profiles are treated as the prediction, and the actual load and PV profiles are generated assuming the value at each step follows a normal distribution with a mean equal to predicted value and a specified standard deviation. Because our prediction horizon is only one day, we can reasonably assume low prediction errors [6], especially for the PV generation. In this case, we test two sets of standard deviations $(\sigma)$ : 1) $\sigma_{P V \text {, load }}=5,15 \%$, and 2) $\sigma_{P V, \text { load }}=10,30 \%$. In order to maintain the locational similarity among all the prosumers in each model run, only one actual PV profile is generated and used for all PV systems, whereas the actual load profiles are generated separately for each individual prosumer. In each model run, the actual coalitional energy cost savings is calculated by applying the ES operations optimized using the predicted profiles to the actual profiles. We then compare the change in coalitional energy cost savings to the predicted value.

For each set of uncertainty inputs, we run the model 200 times with different numbers $(N=6,8,10,12)$ of players. We graph the average change in coalitional energy cost savings as a percentage of the predicted value in Fig. 6. It is shown that prediction errors can lead to reduction in coalitional energy cost savings, but the reduction is relatively small, $2-3 \%$ for the low uncertainty case, and $4-5 \%$ for the high uncertainty case. The change is made less significant because both the individual players' energy costs and the coalitional energy cost are affected by the uncertainties in the same direction, which in turn cancel each other out to an extent when the difference between the two is taken to calculate the cost savings. Additionally, this small reduction remains at a similar level as the number of players grows. This shows that even 


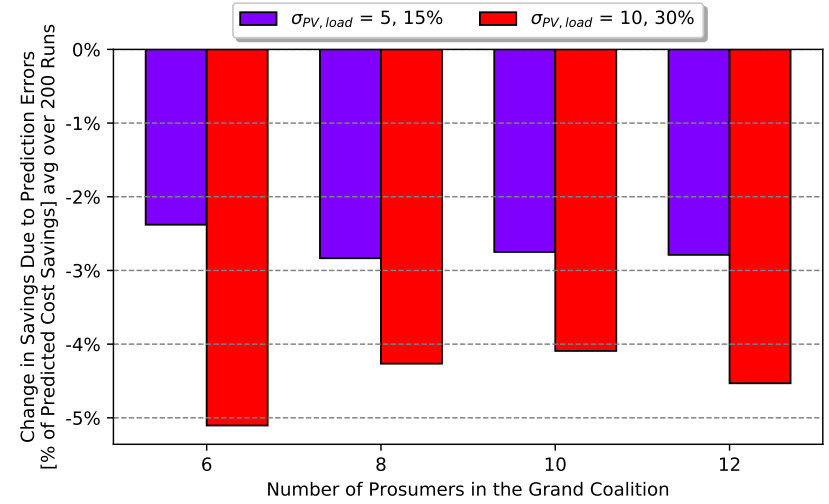

Fig. 6: Uncertainty induced change in coalitional energy cost savings as a percentage of the predicted value by number of players

with uncertainty, the strategy still allows the players to be made significantly better off than they would be on their own.

\section{CONCLUSION}

Using a cooperative game theoretic approach, we propose a prosumer energy grand coalition, which manages all the ES systems within the coalition in a collaborative manner. This cooperative approach not only financially rewards all the participating prosumers, but also benefits the electricity distribution network by reducing the reverse power flow and flattening the local energy profile. Adapting existing ES control algorithms, we formulated a linear problem to optimize the simultaneous operations of multiple ES systems. The values of all possible coalitions within the grand coalition are defined by the coalitional energy cost savings, which can be quantified using the proposed ES optimization algorithm. We proved this prosumer cooperative game to be balanced, meaning the nucleolus, a way to allocate payoffs, is in the core, which guarantees that all players are not only financially rewarded, but also disincentivized from leaving the grand coalition to form smaller coalitions.

Our case study results verify that this coalitional structure helps mitigate the stress on the local power network, and that the nucleolus offers payoffs at a level where no prosumers can benefit from leaving the grand coalition. A key limitation is the computational complexity which increases exponentially with the number of prosumers. Importantly, it has been shown that stabilizing allocations exist for cooperative ES optimization among groups of prosumers, and the stabilizing allocations given by the nucleolus provide an important baseline to assess other allocation methods. The development of sampling algorithms for the nucleolus calculation to reduce the computational burden is a promising area for future work. Additionally, due to the generation and consumption prediction inaccuracy, a small reduction in the actual coalitional energy cost savings is presented. Improving prediction accuracy and developing algorithms to distribute this savings reduction to prosumers while maintaining the stabilizing feature of the nucleolus is also an interesting extension of the current scheme.

\section{APPENDix A \\ PROOF OF LEMMA 1}

When $G\left(\mathfrak{B}^{\langle\mathcal{S}\rangle}, \mathbf{E}^{\mathcal{S}}, \underline{\mathbf{B}}^{\mathcal{S}}, \overline{\mathbf{B}}^{\mathcal{S}}\right)=1$, based on definition, we have (10), (11) and (12) satisfied. Noticing the linearity of these constraints, we can scale them by a factor of $a>0$ :

1) Power constraint:

$$
a \underline{\mathbf{B}}^{\mathcal{S}} \leq a \mathfrak{B}^{\langle\mathcal{S}\rangle} \leq a \overline{\mathbf{B}}^{\mathcal{S}}
$$

2) Energy constraint:

$$
\begin{aligned}
& a \mathbf{E}^{\mathcal{S}} \underline{\mathbf{S o C}} \\
\leq & a \mathbf{E}^{\mathcal{S}} \overline{\mathbf{S o C}^{\mathbf{0}}}+\mathbf{A}^{K}\left(\left[a \mathfrak{B}^{\langle\mathcal{S}\rangle}\right]^{+} \eta^{\mathbf{I}}+\left[a \mathfrak{B}^{\langle\mathcal{S}\rangle}\right]^{-} \eta^{\mathbf{O}}\right) \\
\leq & a \mathbf{E}^{\mathcal{S}} \overline{\mathbf{S o C}}
\end{aligned}
$$

3) Cycle constraint:

$$
\left(\mathbf{1}^{K}\right)^{\boldsymbol{\top}}\left(\left[a \mathfrak{B}^{\langle\mathcal{S}\rangle}\right]^{+} \eta^{\mathbf{I}}+\left[a \mathfrak{B}^{\langle\mathcal{S}\rangle}\right]^{-} \eta^{\mathbf{O}}\right)=\mathbf{0}
$$

Therefore, $G\left(a \mathfrak{B}^{\langle\mathcal{S}\rangle}, a \mathbf{E}^{\mathcal{S}}, a \underline{\mathbf{B}}^{\mathcal{S}}, a \overline{\mathbf{B}}^{\mathcal{S}}\right)=1$

\section{APPENDIX B}

Proof OF LEMMA 2

For $\mathcal{S} \cap \mathcal{T}=\emptyset$, we have

$$
\begin{aligned}
\mathfrak{O}_{\mathcal{S}+\mathcal{T}} & =\left\{\mathfrak{B} \in \mathbb{R}^{K \times N} \mid G\left(\mathfrak{B}, \mathbf{E}^{\mathcal{S}+\mathcal{T}}, \underline{\mathbf{B}}^{\mathcal{S}+\mathcal{T}}, \overline{\mathbf{B}}^{\mathcal{S}+\mathcal{T}}\right)=1\right\} \\
& \stackrel{(7)}{=}\left\{\mathfrak{B} \in \mathbb{R}^{K \times N} \mid G\left(\mathfrak{B}, \mathbf{E}^{\mathcal{S} \cup \mathcal{T}}, \underline{\mathbf{B}}^{\mathcal{S} \cup \mathcal{T}}, \overline{\mathbf{B}}^{\mathcal{S} \cup \mathcal{T}}\right)=1\right\} \\
& =\mathfrak{O}_{\mathcal{S} \cup \mathcal{T}}
\end{aligned}
$$

$\mathcal{S}$ can be considered the union of all its players, and because the intersection of any two different players is $\emptyset$, we have

$$
\begin{aligned}
& \mathbf{Q}^{\mathcal{S}}=\mathbf{Q}^{\bigcup_{i \in \mathcal{S}}\{i\}} \stackrel{\stackrel{(7)}{=}}{=} \mathbf{Q}^{\sum_{i \in \mathcal{S}}\{i\}}=\mathbf{Q}^{\sum_{i \in \mathcal{N}} 1_{i}^{\mathcal{S}}\{i\}} \\
& \mathfrak{O}_{\mathcal{S}}=\mathfrak{O}_{\bigcup_{i \in \mathcal{S}}\{i\}} \stackrel{(34)}{=} \mathfrak{O}_{\sum_{i \in \mathcal{S}}\{i\}}=\mathfrak{O}_{\sum_{i \in \mathcal{N}} 1_{i}^{\mathcal{S}}\{i\}}
\end{aligned}
$$

where $\mathbf{Z} \in \mathbb{R}^{K \times N}$. Therefore,

$$
\begin{aligned}
\theta\left[\sum_{j=1}^{2^{N}} a_{j} \mathcal{S}_{j}\right] & =\min _{\mathfrak{B} \in \mathcal{D}} F\left(\mathfrak{B}, \mathbf{Q}^{\sum_{j=1}^{2_{j}^{N}} a_{j} \mathcal{S}_{j}} a_{j} \mathcal{S}_{j}\right) \\
& \stackrel{(6)(35)}{=} \min _{\mathfrak{B} \in \mathfrak{D}} F\left(\mathfrak{B}, \mathbf{Q}^{\sum_{j=1}^{2_{j=1}^{N}} a_{j} \sum_{j} \sum_{i \in \mathcal{N}} 1_{i} \mathcal{S}_{j}}\{i\}\right) \\
& =\theta\left[\sum_{j=1}^{\mathcal{S}_{j}} a_{i} a_{j} \sum_{i \in \mathcal{N}} 1_{i}^{\mathcal{S}_{j}}\{i\}\right]=\theta\left[\sum_{i \in \mathcal{N}} \sum_{j=1}^{2^{N}} a_{j} 1_{i}^{\mathcal{S}_{j}}\{i\}\right]
\end{aligned}
$$

APPENDiX C

PROOF OF LEMMA 3

\section{A. Proof of Positivity Homogeneity}

When $a=0$, we have

$$
\begin{aligned}
& \quad \theta[a \mathcal{S}]=\theta[0 \mathcal{S}] \\
& \stackrel{(13)}{=} \min _{\mathfrak{B} \in \mathbb{R}^{K \times N}} F\left(\mathfrak{B}, \mathbf{Q}^{0 \mathcal{S}}\right) \quad \text { s.t. } G\left(\mathfrak{B}, \mathbf{E}^{0 \mathcal{S}}, \underline{\mathbf{B}}^{0 \mathcal{S}}, \overline{\mathbf{B}}^{0 \mathcal{S}}\right)=1 \\
& \stackrel{(4)}{=} \min _{\mathfrak{B} \in \mathbb{R}^{K \times N}} F\left(\mathfrak{B}, 0 \mathbf{Q}^{\mathcal{S}}\right) \quad \text { s.t. } G\left(\mathfrak{B}, 0 \mathbf{E}^{\mathcal{S}}, 0 \underline{\mathbf{B}}^{\mathcal{S}}, 0 \overline{\mathbf{B}}^{\mathcal{S}}\right)=1 \\
& =F(\mathbf{0}, \mathbf{0})=0=0 \theta[\mathcal{S}]=a \theta[\mathcal{S}]
\end{aligned}
$$

When $a>0$, we have

$$
C_{a \mathcal{S}}\left(\mathfrak{B}^{\langle a \mathcal{S}\rangle *}\right) \leq C_{a \mathcal{S}}\left(\mathfrak{B}^{\langle a \mathcal{S}\rangle}\right), \forall \mathfrak{B}^{\langle a \mathcal{S}\rangle} \in \mathfrak{O}_{a \mathcal{S}}
$$

Because $\mathfrak{B}^{\langle a \mathcal{S}\rangle *} \in \mathfrak{O}_{a \mathcal{S}}$, we have

$$
G\left(\mathfrak{B}^{\langle a \mathcal{S}\rangle *}, \mathbf{E}^{a \mathcal{S}}, \underline{\mathbf{B}}^{a \mathcal{S}}, \overline{\mathbf{B}}^{a \mathcal{S}}\right)=1
$$

We define $\mathfrak{B}^{\langle\mathcal{S}\rangle^{\prime}}=\frac{1}{a} \mathfrak{B}^{\langle a \mathcal{S}\rangle *}$. Using Lemma 1, we have

Using (4), we get

$$
G\left(\mathfrak{B}^{\langle\mathcal{S}\rangle^{\prime}}, \frac{1}{a} \mathbf{E}^{a \mathcal{S}}, \frac{1}{a} \underline{\mathbf{B}}^{a \mathcal{S}}, \frac{1}{a} \overline{\mathbf{B}}^{a \mathcal{S}}\right)=1
$$

$$
G\left(\mathfrak{B}^{\langle\mathcal{S}\rangle^{\prime}}, \mathbf{E}^{\mathcal{S}}, \underline{\mathbf{B}}^{\mathcal{S}}, \overline{\mathbf{B}}^{\mathcal{S}}\right)=1
$$


In other words, $\mathfrak{B}^{\langle\mathcal{S}\rangle^{\prime}} \in \mathfrak{O}_{\mathcal{S}}$. We then have

$$
\begin{aligned}
& \theta[\mathcal{S}] \stackrel{(14)}{=} C_{\mathcal{S}}\left(\mathfrak{B}^{\langle\mathcal{S}\rangle *}\right) \stackrel{(15)}{\leq} C_{\mathcal{S}}\left(\mathfrak{B}^{\langle\mathcal{S}\rangle^{\prime}}\right) \\
& \stackrel{(9)}{=} F\left(\frac{1}{a} \mathfrak{B}^{\langle a \mathcal{S}\rangle *}, \mathbf{Q}^{\mathcal{S}}\right)=F\left(\frac{1}{a} \mathfrak{B}^{\langle a \mathcal{S}\rangle *}, \frac{1}{a} \mathbf{Q}^{a \mathcal{S}}\right) \\
& \stackrel{(2)}{=} \frac{1}{a} F\left(\mathfrak{B}^{\langle a \mathcal{S}\rangle *}, \mathbf{Q}^{a \mathcal{S}}\right) \stackrel{(9)}{=} \frac{1}{a} C_{a \mathcal{S}}\left(\mathfrak{B}^{\langle a \mathcal{S}\rangle *}\right) \stackrel{(14)}{=} \frac{1}{a} \theta[a \mathcal{S}]
\end{aligned}
$$

After rearrangement, we have

$$
\theta[a \mathcal{S}] \geq a \theta[\mathcal{S}], \forall a>0
$$

Since $a$ can be any positive value, we construct

$$
\theta[\mathcal{S}]=\theta\left[\frac{1}{a}(a \mathcal{S})\right]
$$

Since $\frac{1}{a}>0$, we have

$$
\theta[\mathcal{S}]=\theta\left[\frac{1}{a}(a \mathcal{S})\right] \geq \frac{1}{a} \theta[a \mathcal{S}]
$$

Combining the equations above, We have

$$
\theta[a \mathcal{S}] \geq a \theta[\mathcal{S}] \geq a \frac{1}{a} \theta[a \mathcal{S}]=\theta[a \mathcal{S}]
$$

Therefore, it can only be the case that

$$
\theta[a \mathcal{S}]=a \theta[\mathcal{S}]
$$

\section{B. Proof of Subadditivity}

We rewrite (1) with variable $\mathbf{L}=\mathfrak{B}+\mathbf{Q}, \forall \mathfrak{B}, \mathbf{Q} \in \mathbb{R}^{K \times N}$ :

$$
F(\mathbf{L})=\mathbf{p}_{b}^{\top}\left[\mathbf{L} 1^{N}\right]^{+}+\mathbf{p}_{s}^{\top}\left[\mathbf{L} 1^{N}\right]^{-}
$$

For any $\mathbf{L}_{1}, \mathbf{L}_{2} \in \mathbb{R}^{K \times N}, \mathbf{p}_{b}^{\top} \geq \mathbf{p}_{s}^{\top}$, and $a \geq 0$, we have

1) (Positive Homogeneity) $F\left(a \mathbf{L}_{1}\right)=a F\left(\mathbf{L}_{1}\right)$

2) (Convexity) $\frac{F\left(\mathbf{L}_{1}\right)+F\left(\mathbf{L}_{2}\right)}{2} \geq F\left(\frac{\mathbf{L}_{1}+\mathbf{L}_{2}}{2}\right)$

We have $F\left(\mathbf{L}_{1}\right)+F\left(\mathbf{L}_{2}\right)$

$$
\begin{aligned}
& \geq 2 F\left(\frac{\mathbf{L}_{1}+\mathbf{L}_{2}}{2}\right), \text { by convexity of } F \\
& =F\left(\mathbf{L}_{1}+\mathbf{L}_{1}\right), \text { by positive homogeneity of } F
\end{aligned}
$$

If we denote $\mathbf{L}^{\langle a \mathcal{S}\rangle *}=\mathfrak{B}^{\langle a \mathcal{S}\rangle *}+\mathbf{Q}^{a \mathcal{S}}, \forall \mathcal{S} \in 2^{\mathcal{N}}$, we have

$$
\theta[a \mathcal{S}]+\theta[b \mathcal{T}]=F\left(\mathbf{L}^{\langle a \mathcal{S}\rangle *}\right)+F\left(\mathbf{L}^{\langle b \mathcal{T}\rangle *}\right)
$$

$$
\begin{aligned}
& \stackrel{(36)}{\geq} F\left(\mathbf{L}^{\langle a \mathcal{S}\rangle *}+\mathbf{L}^{\langle b \mathcal{T}\rangle *}\right) \\
& =F\left(\mathfrak{B}^{\langle a \mathcal{S}\rangle *}+\mathfrak{B}^{\langle b \mathcal{T}\rangle *}, \mathbf{Q}^{a \mathcal{S}}+\mathbf{Q}^{b \mathcal{T}}\right) \\
& \stackrel{(6)}{=} C_{a \mathcal{S}+b \mathcal{T}}\left(\mathfrak{B}^{\langle a \mathcal{S}\rangle *}+\mathfrak{B}^{\langle b \mathcal{T}\rangle *}\right)
\end{aligned}
$$

We know $\left\{\begin{array}{l}G\left(\mathfrak{B}^{\langle a \mathcal{S}\rangle *}, \mathbf{E}^{a \mathcal{S}}, \underline{\mathbf{B}}^{a \mathcal{S}}, \overline{\mathbf{B}}^{a \mathcal{S}}\right)=1 \\ G\left(\mathfrak{B}^{\langle b \mathcal{T}\rangle *}, \mathbf{E}^{b \mathcal{T}}, \underline{\mathbf{B}}^{b \mathcal{T}}, \overline{\mathbf{B}}^{b \mathcal{T}}\right)=1\end{array}\right.$

Because $\eta^{\mathbf{I}}, \eta^{\mathbf{O}} \leq \mathbf{1}$, by combining the ES energy capacities, charge limits and discharge limits using (6) we can construct $\mathfrak{B}^{\langle a \mathcal{S}+b \mathcal{T}\rangle}$ such that

$$
\begin{aligned}
& C_{a \mathcal{S}+b \mathcal{T}}\left(\mathfrak{B}^{\langle a \mathcal{S}\rangle *}+\mathfrak{B}^{\langle b \mathcal{T}\rangle *}\right) \geq C_{a \mathcal{S}+b \mathcal{T}}\left(\mathfrak{B}^{\langle a \mathcal{S}+b \mathcal{T}\rangle}\right), \\
& G\left(\mathfrak{B}^{\langle a \mathcal{S}+b \mathcal{T}\rangle}, \mathbf{E}^{a \mathcal{S}+b \mathcal{T}}, \underline{\mathbf{B}}^{a \mathcal{S}+b \mathcal{T}}, \overline{\mathbf{B}}^{a \mathcal{S}+b \mathcal{T}}\right)=1
\end{aligned}
$$

Based on (15), we have

$$
C_{a \mathcal{S}+b \mathcal{T}}\left(\mathfrak{B}^{\langle a \mathcal{S}+b \mathcal{T}\rangle}\right) \geq C_{a \mathcal{S}+b \mathcal{T}}\left(\mathfrak{B}^{\langle a \mathcal{S}+b \mathcal{T}\rangle *}\right)
$$

Combining (37), (38) and (39), we prove

$$
\theta[a \mathcal{S}]+\theta[b \mathcal{T}] \geq C_{a \mathcal{S}+b \mathcal{T}}\left(\mathfrak{B}^{\langle a \mathcal{S}+b \mathcal{T}\rangle *}\right)=\theta[a \mathcal{S}+b \mathcal{T}]
$$

\section{APPENDIX D}

PROOF OF THEOREM 1

Using (16), we can rewrite the left-hand side of (18):

$$
\begin{aligned}
& \sum_{\mathcal{S} \in 2^{\mathcal{N}}} \alpha(\mathcal{S}) v(\mathcal{S}) \\
= & \sum_{\mathcal{S} \in 2^{\mathcal{N}}} \alpha(\mathcal{S})\left[\sum_{i \in \mathcal{S}} C_{\{i\}}\left(\mathfrak{B}^{\langle\{i\}\rangle *}\right)-C_{\mathcal{S}}\left(\mathfrak{B}^{\langle\mathcal{S}\rangle *}\right)\right] \\
= & \sum_{i \in \mathcal{N}} \sum_{\mathcal{S} \in 2^{\mathcal{N}}} \alpha(\mathcal{S}) 1_{i}^{\mathcal{S}} C_{\{i\}}\left(\mathfrak{B}^{\langle\{i\}\rangle *}\right)-\sum_{\mathcal{S} \in 2^{\mathcal{N}}} \alpha(\mathcal{S}) C_{\mathcal{S}}\left(\mathfrak{B}^{\langle\mathcal{S}\rangle *}\right) \\
= & \sum_{i \in \mathcal{N}} C_{\{i\}}\left(\mathfrak{B}^{\langle\{i\}\rangle *}\right)-\sum_{\mathcal{S} \in 2^{\mathcal{N}}} \alpha(\mathcal{S}) C_{\mathcal{S}}\left(\mathfrak{B}^{\langle\mathcal{S}\rangle *}\right)
\end{aligned}
$$

and the right-hand side of (18):

$$
v(\mathcal{N})=\sum_{i \in \mathcal{N}} C_{\{i\}}\left(\mathfrak{B}^{\langle\{i\}\rangle *}\right)-C_{\mathcal{N}}\left(\mathfrak{B}^{\langle\mathcal{N}\rangle *}\right)
$$

Combining (41) and (42), we can see that our cooperative game is balanced as long as for any balanced map $\alpha$,

$$
C_{\mathcal{N}}\left(\mathfrak{B}^{\langle\mathcal{N}\rangle *}\right) \leq \sum_{\mathcal{S} \in 2^{\mathcal{N}}} \alpha(\mathcal{S}) C_{\mathcal{S}}\left(\mathfrak{B}^{\langle\mathcal{S}\rangle *}\right)
$$

Rewriting (43) using (14), we only need to prove

$$
\theta[\mathcal{N}] \leq \sum_{\mathcal{S} \in 2^{\mathcal{N}}} \alpha(\mathcal{S}) \theta[\mathcal{S}], \forall \alpha: 2^{\mathcal{N}} \rightarrow[0,1]
$$

We then have

$$
\begin{aligned}
& \sum_{\mathcal{S} \in 2^{\mathcal{N}}} \alpha(\mathcal{S}) \theta[\mathcal{S}] \\
= & \sum_{\mathcal{S} \in 2^{\mathcal{N}}} \theta[\alpha(\mathcal{S}) \mathcal{S}], \quad \text { by positive homogeneity of } \theta \\
\geq & \theta\left[\sum_{\mathcal{S} \in 2^{\mathcal{N}}} \alpha(\mathcal{S}) \mathcal{S}\right], \quad \text { by subadditivity of } \theta \\
= & \theta\left[\sum_{i \in \mathcal{N}} \sum_{\mathcal{S} \in 2^{\mathcal{N}}} \alpha(\mathcal{S}) 1_{i}^{\mathcal{S}}\{i\}\right], \quad \text { by Lemma } 2 \\
= & \theta\left[\sum_{i \in \mathcal{N}}\{i\}\right]=\theta[\mathcal{N}], \quad \text { by } \alpha(\mathcal{S}) \text { definition, Lemma } 2
\end{aligned}
$$

Hence (44) is proven, further proving the game is balanced.

\section{REFERENCES}

[1] S. Bull, "Renewable energy today and tomorrow," Proceedings of the IEEE, vol. 89, no. 8, pp. 1216-1226, 2001.

[2] P. H. J. Skea, D. Anderson, T. Green, R. Gross and M. Leach, "Intermittent renewable generation and the cost of maintaining power system reliability," Generation, Transmission \& Distribution, IET, vol. 1, no. 2, p. 324, 2007.

[3] C. Masters, "Voltage rise: the big issue when connecting embedded generation to long $11 \mathrm{kV}$ overhead lines," Power Engineering Journal, vol. 16, no. 1, pp. 5-12, 2002.

[4] K. C. Divya and J. Østergaard, "Battery energy storage technology for power systems-An overview," Electric Power Systems Research, vol. 79 , no. 4, pp. 511-520, 2009.

[5] T. Morstyn, B. Hredzak, and V. G. Agelidis, "Control strategies for microgrids with distributed energy storage systems: An overview," IEEE Transactions on Smart Grid, vol. PP, no. 99, pp. 1-1, 2016.

[6] K. Worthmann, C. M. Kellett, P. Braun, L. Grüne, and S. R. Weller, "Distributed and Decentralized Control of Residential Energy Systems Incorporating Battery Storage," IEEE Transactions on Smart Grid, vol. 6, no. 4, pp. 1914-1923, 2015.

[7] Y. Gu, X. Xiang, W. Li, and X. He, "Mode-adaptive decentralized control for renewable dc microgrid with enhanced reliability and flexibility," IEEE Transactions on Power Electronics, vol. 29, no. 9, pp. 5072-5080, Sept 2014.

[8] Y. S. F. Eddy, H. B. Gooi, and S. X. Chen, "Multi-agent system for distributed management of microgrids," IEEE Transactions on Power Systems, vol. 30, no. 1, pp. 24-34, Jan 2015. 
[9] T. Morstyn, B. Hredzak, and V. G. Agelidis, "Distributed cooperative control of microgrid storage," IEEE Transactions on Power Systems, vol. 30, no. 5, pp. 2780-2789, Sept 2015.

[10] M. Liu, P. K. Phanivong, and D. S. Callaway, "Electric vehicle charging control in residential distribution network: A decentralized event-driven realization," in 2017 IEEE 56th Annual Conference on Decision and Control (CDC), Dec 2017, pp. 214-219.

[11] T. Morstyn, B. Hredzak, R. P. Aguilera, and V. G. Agelidis, "Model predictive control for distributed microgrid battery energy storage systems," IEEE Transactions on Control Systems Technology, vol. 26, no. 3, pp. 1107-1114, May 2018.

[12] Y. Wang, "Behavioral Game Theory for Smart Grid Energy Management," Open Access Dissertations, 2015. [Online]. Available: http://scholarlyrepository.miami.edu/oa_dissertations/1377

[13] D. S. Callaway and I. A. Hiskens, "Achieving Controllability of Electric Loads," Proceedings of the IEEE, vol. 99, no. 1, 2010.

[14] P. Berenbrink, T. Friedetzky, L. A. Goldberg, P. W. Goldberg, Z. Hu, and R. Martin, "Distributed selfish load balancing," SIAM Journal on Computing, vol. 37, no. 4, pp. 1163-1181, 2007.

[15] L. Deori, K. Margellos, and M. Prandini, "On the connection between Nash equilibria and social optima in electric vehicle charging control games," 20th World Congress of the International Federation of Automatic Control, 2017.

[16] Z. J. Ma, D. S. Callaway, and I. A. Hiskens, "Decentralized Charging Control of Large Populations of Plug-in Electric Vehicles," IEEE Transactions on Control Systems Technology, vol. 21, no. 1, pp. 67-78, 2013.

[17] B. G. Kim, S. Ren, M. Van Der Schaar, and J. W. Lee, "Bidirectional energy trading for residential load scheduling and electric vehicles," Proceedings - IEEE INFOCOM, vol. 31, no. 7, pp. 595-599, 2013.

[18] L. Jia and L. Tong, "Dynamic pricing and distributed energy management for demand response," IEEE Transactions on Smart Grid, vol. 7, no. 2, pp. 1128-1136, March 2016.

[19] N. Liu, X. Yu, C. Wang, and J. Wang, "Energy sharing management for microgrids with pv prosumers: A stackelberg game approach," IEEE Transactions on Industrial Informatics, vol. 13, no. 3, pp. 1088-1098, June 2017.

[20] M. Yazdani-Damavandi, N. Neyestani, M. Shafie-khah, J. Contreras, and J. P. S. Catalo, "Strategic behavior of multi-energy players in electricity markets as aggregators of demand side resources using a bilevel approach," IEEE Transactions on Power Systems, vol. 33, no. 1, pp. 397-411, Jan 2018.

[21] T. Morstyn, A. Teytelboym, and M. D. McCulloch, "Bilateral contract networks for peer-to-peer energy trading," IEEE Transactions on Smart Grid, pp. 1-1, 2018.

[22] O. Okpako, H. S. Rajamani, P. Pillai, U. Anuebunwa, and K. S. Swarup, "Investigation of an optimized energy resource allocation algorithm for a community based virtual power plant," in 2016 IEEE PES PowerAfrica, June 2016, pp. 153-157.

[23] N. Liu, X. Yu, C. Wang, C. Li, L. Ma, and J. Lei, "Energy-sharing model with price-based demand response for microgrids of peer-to-peer prosumers," IEEE Transactions on Power Systems, vol. 32, no. 5, pp. 3569-3583, Sept 2017.

[24] J. Wang and N. Liu, "Distributed optimal scheduling for multi-prosumers with chp and heat storage based on admm," in IECON 2017 - 43rd Annual Conference of the IEEE Industrial Electronics Society, Oct 2017, pp. 2455-2460.

[25] W. Saad, Z. Han, H. V. Poor, and T. Basar, "Game-theoretic methods for the smart grid: An overview of microgrid systems, demand-side management, and smart grid communications," IEEE Signal Processing Magazine, vol. 29, no. 5, pp. 86-105, Sept 2012.

[26] W. Saad, Z. Han, and H. V. Poor, "Coalitional game theory for cooperative micro-grid distribution networks," IEEE International Conference on Communications, pp. 6-10, 2011.

[27] E. Baeyens, E. Y. Bitar, P. P. Khargonekar, and K. Poolla, "Coalitional aggregation of wind power," IEEE Transactions on Power Systems, vol. 28, no. 4, pp. 3774-3784, 2013.

[28] W. Lee, L. Xiang, R. Schober, and V. W. S. Wong, "Direct electricity trading in smart grid: A coalitional game analysis," IEEE Journal on Selected Areas in Communications, vol. 32, no. 7, pp. 1398-1411, 2014

[29] GOV.UK. (2017, June) Annual domestic energy bills. [Online]. Available: https://www.gov.uk/government/statistical-data-sets/annualdomestic-energy-price-statistics [Accessed: 2017-08-03].

[30] GOV.UK. (2016, Feb.) Feed-in tariffs: get money for generating your own electricity. [Online]. Available: https://www.gov.uk/feed-intariffs/overview [Accessed: 2017-08-03].
[31] H. Wirth, "Recent facts about photovoltaics in Germany," Fraunhofer ISE, p. 92, 2015.

[32] L. Barth, N. Ludwig, E. Mengelkamp, and P. Staudt, "A comprehensive modelling framework for demand side flexibility in smart grids," Computer Science - Research and Development, vol. 33, no. 1, pp. 13-23, Feb 2018.

[33] L. S. Shapley, "Cores of convex games," International Journal of Game Theory, vol. 1, no. 1, pp. 11-26, 1971.

[34] L. Shapley, On Balanced Sets and Cores, ser. Memorandum (Rand Corporation). Rand Corporation, 1965.

[35] T. S. H. Driessen, Cooperative Games, Solutions and Applications, ser. Theory and decision library: Series C, Game theory, mathematical programming, and operations research. Springer, 1988.

[36] M. Gulin, J. Matusko, and M. Vasak, "Stochastic model predictive control for optimal economic operation of a residential DC microgrid," IEEE International Conference on Industrial Technology (ICIT), pp. 505-510, 2015.

[37] E. G. L. Parisio A.; Rikos, "A Model Predictive Control Approach to Microgrid Operation Optimization," Control Systems Technology, IEEE Transactions, vol. 1, no. 4, pp. 1813-1827, 2014.

[38] E. Perez, H. Beltran, N. Aparicio, and P. Rodriguez, "Predictive power control for PV plants with energy storage," IEEE Transactions on Sustainable Energy, vol. 4, no. 2, pp. 482-490, 2013.

[39] Network-Revolution. (2014) Enhanced profiling of domestic customers with solar photovoltaics (pv). [Online] Available: http://www.networkrevolution.co.uk/project-library/datasettc5-enhanced-profiling-solar-photovoltaic-pv-users/ [Accessed: 201710-03].

[40] Pvwatts.nrel.gov. Pvwatts calculator. [Online]. Available: http://pvwatts.nrel.gov/pvwatts.php [Accessed: 2017-08-03]

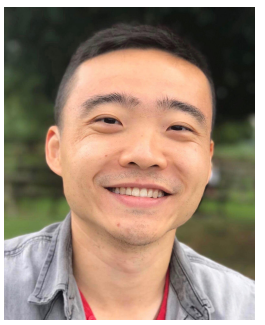

Liyang Han (S'17) received the B.S degree in energy engineering from Zhejiang University, China, in 2012, and the M.S. degree in civil engineering from Stanford University, CA, USA, in 2014.

$\mathrm{He}$ worked in California for two years, first as an Energy Analyst for Integral Group, Inc. and then as an Innovation Energy Services Analyst for EDF, Inc. $\mathrm{He}$ is currently working towards the Ph.D degree at the University of Oxford, UK. His research interests include energy storage dispatch optimization and game theory application in energy networks.

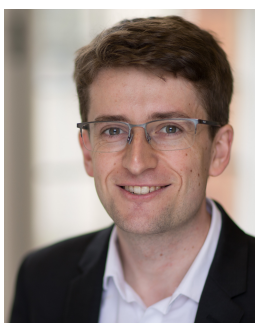

Thomas Morstyn (S'14-M'16) received the B.E. (Hon.) degree from the University of Melbourne, Australia, in 2011, and the $\mathrm{PhD}$ degree from the University of New South Wales, Australia, in 2016, both in electrical engineering.

$\mathrm{He}$ worked as an electrical engineer in the Rio Tinto Technology and Innovation group between 2012 and 2014. He is currently an EPSRC Fellow with the Department of Engineering Science at the University of Oxford. His research interests include multi-agent control and market design for coordinating distributed energy resources in power systems.

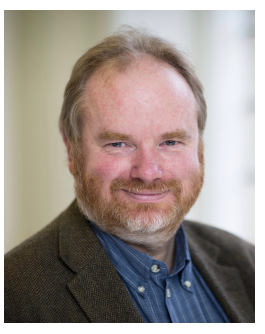

Malcolm D. McCulloch (SM'89) received the B.Sc. (Eng.) and Ph.D. degrees in electrical engineering from the University of the Witwatersrand, Johannesburg, South Africa, in 1986 and 1990, respectively.

In 1993, he joined the University of Oxford, Oxford, U.K., to head up the Energy and Power Group, where he is currently an Associate Professor in the Department of Engineering Science. He is active in the areas of electrical machines, transport, and smart grids. His work addresses transforming existing power networks, designing new power networks for the developing world, developing new technology for electric vehicles, and developing approaches to integrated mobility. 